\title{
Health Insurance Literacy and Health Disparities in the United States: A Literature Review
}

\author{
Emily Vardell, Emporia State University, USA
}

\begin{abstract}
This paper presents a literature review of health insurance literacy with a focus on specialized populations in the U.S. and how limited health literacy skills exacerbate health disparities. This discussion places this issue within the context of contemporary U.S. health care reform and makes connections between health insurance coverage and health disparities. This overview of the research on health insurance literacy covers research across the health insurance spectrum, from awareness of health insurance options to assessments of health literacy skills in specific populations as well as from readability of health insurance informational materials to the availability of multilingual services. In exploring the demographic variables associated with lower health insurance literacy skills, this paper reviews the body of current research in this area to make connections between populations more likely to have unequal access to health care and how having limited skills in navigating the U.S. health care system may compound these disparities. In addition, this paper proposes an Integrated Framework for Health Insurance Literacy as a method for further studying the connections between demographic factors, health coverage, health status, and health insurance literacy skills.
\end{abstract}

Keywords: health disparities; health information; health insurance literacy; health justice

Publication Type: review article

\section{Background}

$\mathrm{n}$ the U.S., private health insurance is the most common mechanism by which individuals protect themselves from the tremendously high costs of medical care due to severe illness or accident (Kaiser Family Foundation, 2008). When an individual has health insurance, the person can use this coverage to subsidize the cost of a visit to their physician. In the case of a physician visit, the insurance company will pay a portion of the cost (sometimes dependent on whether the physician is in the insurance company's preferred network) and the individual will pay a remaining portion (i.e., a copay). Employer-sponsored insurance (ESI) is the most common way individuals are insured in the U.S., with 150 million Americans obtaining employer-sponsored coverage each year (Kaiser Family Foundation, 2016). ESI is an economically effective system in that it allows companies to have a diverse group of people (often of mixed ages and health statuses) and provides a convenient risk pool. This reduces administrative costs and allows employees to cancel out each other's risk.

Health care coverage in the U.S. is unique to the country; most other industrialized nations have a national health care system with universal coverage. For example, in the United Kingdom, coverage is universal with the majority of funding coming from general taxation and a smaller amount coming from a payroll tax for national insurance. Approximately $10.5 \%$ of the population

The International Journal of Information, Diversity, \& Inclusion, 3(4), 2019

ISSN 2574-3430, jps.library.utoronto.ca/index.php/ijidi/index

DOI: $10.33137 /$ ijidi.v3i4.33012 
in the U.K. also has private voluntary insurance, seen as a method for obtaining more rapid access to care (Thorlby \& Arora, 2019). Similarly, Germany has mandatory health coverage with competing, not-for-profit, nongovernmental health insurance funds options as well as private health insurance. In Japan, citizens are covered with a universal Statutory Health Insurance System with a majority of the population also obtaining private health insurance in a supplementary capacity. Israel's National Health Insurance system automatically covers citizens and permanent residents, all of whom select from four competing, nonprofit plans.

Each year, Americans with ESI must select their preferred insurance coverage option from a handful of choices made available to them through their employers. These decisions are often made with only Summary of Benefits and Coverage (SBC) forms as guides. SBC forms are designed to provide standardized information about different options to enable employees to select their optimal option. Assessments of health insurance informational materials have demonstrated high literacy demands (Pati et al., 2012; Vardell, 2013), indicating that individuals with lower health literacy levels may not have the skills necessary to interpret SBC forms.

In addition, there is a growing trend in U.S. health care to encourage healthy individuals to enroll in consumer-directed health plans. Consumer-directed health plans (CDHPs), or low copay, high deductible plans, require enrollees to compare costs between providers and treatment options. Those who do not have high levels of health insurance literacy may not have the skills to participate effectively in a consumer-directed health plan, leading to potential diminished ability to save on health care costs.

Underpinning health insurance choice research is the idea that individuals should select the health care coverage that matches their anticipated health care utilization. Health insurance companies should, in a fair market, compete to attract subscribers with a financially competitive offering. However, consumers are often unable to select what can be considered their most financially appropriate option given their anticipated health care costs. Consequently, the competition in the market is minimized and "naïve consumers pay prices substantially above marginal cost, and effectively subsidize sophisticated consumers who are able to exploit the mispricing" (Loewenstein et al., 2013, p. 851).

If an individual pays a regular monthly premium for coverage that far exceeds their anticipated cost, they are not getting the maximum value for their money. On the other hand, if an individual selects health insurance coverage that offsets more of the costs to the consumer (an ideal design for those who require minimal health care services) and then uses a high level of health care services, they will pay higher medical bills than if they had selected a coverage option that required higher monthly premiums but lower costs at the point-of-care. Individuals may also demonstrate preferences to pay different types of costs (Vardell, 2017); for example, some individuals demonstrate a preference for paying a deductible over paying a monthly premium, even if from an objective observer a different plan would be more fiscally appropriate.

Employees have been making health insurance decisions for many years, and now the Affordable Care Act (ACA) has brought the issues of health insurance literacy to the spotlight. Through the ACA, millions of previously uninsured persons are making health insurance choices and using health care coverage for the first time, and Americans with employer-sponsored insurance have seen a change in coverage benefits (Patient Protection \& Affordable Care Act, 2010).

While health literacy and its ramifications on understanding general health information have

The International Journal of Information, Diversity, \& Inclusion, 3(4), 2019

ISSN 2574-3430, jps.library.utoronto.ca/index.php/ijidi/index

DOI: $10.33137 /$ ijidi.v3i4.33012 
been studied extensively (Paasche-Orlow, Parker, Gazmararian, Nielsen-Bohlman, \& Rudd, 2005), only a limited amount of research has been focused on health insurance literacy. In fact, though it may seem apparent that many individuals lack clear understanding of their health insurance, it is a "widely perceived but poorly documented problem" (Loewenstein et al., 2013, p. 851). One of the first formally proposed definitions of health insurance literacy describes it as "the extent to which consumers can make informed purchase and use decisions" (Kim, Braun, \& Williams, 2013, p. 3).

For the 77 million adults with basic or below basic health literacy (Kutner, Greenburg, Jin, \& Paulsen, 2006), their ability to procure appropriate levels of health insurance coverage and interact with the health care system successfully may be limited. Initial research in this area has shown that health insurance information materials are not written with low-literacy users in mind (Pati et al., 2012; Vardell, 2013). Patients may have difficulties navigating the intricacies of health insurance demands, such as selecting a physician within the insurance company's preferred network, understanding what types of care are included at no extra cost (e.g., preventive services), and considering using prescription medications that are on the insurance company's preferred list of medications. Participants in Vardell's 2017 study revealed delaying or avoiding care due to high medical care costs and not understanding what coverage was included in their insurance plans.

\section{Connections between Health Insurance Coverage and Health Disparities}

Access to health insurance and health care coverage are vital in addressing health disparities. A study of citizens in Massachusetts demonstrated overall improvements in individuals' selfassessed health following Massachusetts' health care reform which required universal coverage of all citizens and which is an antecedent model for the Affordable Care Act (Courtemanche \& Zapata, 2014). Physical health, mental health, joint disorders, and body mass index were all demonstrated to improve following health care reform. In addition, the improvements were strongest for people with lower incomes, nonwhites, and near-elderly adults, many of whom have been shown to be more greatly affected by health disparities.

This initial study by Courtemanche and Zapata demonstrates promise for health care reform as a method for reducing health care disparities. However, the disconnect between insurance plan literacy demands and the literacy levels of enrollees may increase health disparities and health care costs among a large portion of the population (Miller, 2007). The connection between health care reform and health insurance literacy is highlighted in this quote from the U.S. Department of Health and Human Services (2008): "The success of health system reform will depend in large part on the capacity of individuals, families, and communities to make informed decisions about their health" (p. 7). Health care reform is a significant piece in addressing health disparities, but improving consumer health insurance literacy may be equally essential.

\section{Methods}

To explore the connection between health insurance literacy and health disparities, the author conducted a literature review, which is presented in this paper. Related terms including health insurance, health policy, and health literacy were searched in PubMed, Web of Science, LexisNexis, RAND Publication Database, Business Source Complete, and PAIS International. Articles were reviewed individually to determine relevance. In addition, the author used Web of Knowledge to perform citation chaining of the most relevant articles, identifying additional

The International Journal of Information, Diversity, \& Inclusion, 3(4), 2019

ISSN 2574-3430, jps.library.utoronto.ca/index.php/ijidi/index

DOI: $10.33137 /$ ijidi.v3i4.33012 
articles for inclusion.

\section{Literature Review}

This literature review will discuss literacy concerns across the health insurance process, beginning with awareness of health insurance and followed by a discussion of large-scale assessments of health insurance literacy. A discussion of the literacy demands in the health insurance process, with a focus on the readability of health insurance informational materials and forms, will follow.

\section{Awareness of Health Insurance}

The first step in effective use of the health care system is awareness of the available resources. Federman et al. (2009) conducted a study of inner-city seniors to determine awareness of pharmaceutical cost-assistance programs, such as Medicaid supplemental programs. The researchers interviewed inner-city seniors about their awareness of programs, participation in health insurance presentations, and other demographic factors. Male gender, Black race, inadequate health literacy (measured using the Short Test of Functional Health Literacy in Adults), and receiving care in a clinic setting (as opposed to private or group practice) were associated with low awareness of cost-assistance programs. Study participants who had heard a live presentation about health insurance were more likely to be aware of such programs. The authors suggest that their findings support the "use of live presentations, in addition to health literacy materials and messages, [as] . . . important strategies in promoting knowledge of and enrollment in state and federal pharmaceutical cost-assistance programs for low-income seniors" (p. 127-129). This study underscores the importance of first knowing that programs exist. A lack of knowledge about such programs, particularly among African-Americans and those accessing care in public clinics, could further exacerbate unequal access to health care coverage and access that has already been identified among minority populations (U.S. Department of Health and Human Services, 2001).

\section{Measurements of Health Literacy and Health Insurance Literacy}

In addition to assessing awareness of health insurance, researchers have also assessed health insurance skills using a variety of metrics and approaches outlined in this section. In large studies of health insurance literacy levels, individuals who struggled the most with understanding health care are more likely to be 65 years or older and/or ethnic minorities (Kutner et al., 2006; McCormack, Bann, Uhrig, Berkman, \& Rudd, 2009). These studies also show mixed results in regards to the role of gender in health insurance literacy levels. Additional populations identified as more likely to have lower levels of health literacy levels include those having spoken a language besides English prior to formal education (Kutner et al, 2006), adults from a lower socio-economic level and those who reported a lower health status (McCormack et al., 2009); individuals with lower levels of education, younger Americans, and the uninsured (Norton, Hamel, and Brodie, 2014).

In the 2003 National Assessment of Adult Literacy (NAAL, the largest-scale measurement of health literacy in the United States to date), more than one quarter of the population with employerbased insurance demonstrated little to no "simple and concrete literacy skills" (Kutner et al., 2006, p. 5). While the greatest percentage of adults with employer, military, or private insurance had intermediate or proficient health literacy, individuals with U.S. government-sponsored

The International Journal of Information, Diversity, \& Inclusion, 3(4), 2019

ISSN 2574-3430, jps.library.utoronto.ca/index.php/ijidi/index

DOI: $10.33137 /$ ijidi.v3i4.33012 
Medicare, Medicaid, or no insurance had the greatest percentage of below basic health literacy (more than half of that population had basic or below basic health literacy skills; $25 \%$ had basic health literacy and $28 \%$ had below basic literacy). This statistic demonstrates the compounding nature of health disparities; not only do these individuals have limited access to health care, but they also have lower skill levels for interacting in the system effectively. In addition, individuals who struggle the most with understanding health care information were more likely to be 65 years or older, male, African American or Hispanic, and/or have spoken another language besides English prior to formal education.

McCormack et al. (2009) used principles of financial literacy, coupled with previous research on health literacy, to examine health insurance literacy in their study sample of 1,202 Medicare beneficiaries participating in the longitudinal Medicare Current Beneficiary Survey. Their group created a two-part instrument to assess health insurance literacy, including questions designed to gauge prior knowledge and familiarity with health insurance terminology and questions aimed at assessing proficiency using the Medicare insurance system (as their focus was on older adults).

In the proficiency exercises designed by McCormack et al. (2009), the questions requiring interpretation of the Medicare Explanation of Benefits form proved to be the most difficult. The authors demonstrated that "certain vulnerable subgroups also had significantly lower levels of health insurance literacy relative to their counterparts" (p. 236), including adults over the age of 85 , women, ethnic minorities, adults from a lower socio-economic level, and those who reported a lower health status. A 2005 systematic review of health literacy research corroborates these 2009 findings, as it demonstrated that lower levels of health literacy are associated with levels of education, ethnicity, and age; however, the systematic review also demonstrated that lower health literacy is not associated with gender or measurement instrument (Paasche-Orlow et al., 2005). The relationship between gender and health insurance literacy may require additional research due to conflicting findings, some of which show males with lower health insurance literacy (Federman et al, 2009; Kutner et al., 2006; Politi et al., 2014), while others show women with lower levels (McCormack et al., 2009).

Politi et al. (2014) examined 51 uninsured adults' (mostly low-income and African-American) health insurance literacy and preferences using semi-structured interviews. The participants demonstrated minimal understanding of common health insurance terminology (coinsurance, deductible, out-of-pocket maximum, prior authorization, and formulary were the least familiar terms). Participants with no previous history of health insurance demonstrated lower health insurance literacy than those who had previously been insured. In addition, their study demonstrated that those with lower general health literacy also had poorer understanding of insurance-specific topics.

In an examination of the strategies that participants used to understand health insurance terms, Politi et al. observed that about half of their participants connected concepts from non-health contexts to understand the terminology. For example, participants recognized the term "deductible" from car insurance and "referral" from searching for jobs. These results suggest that providing context around terminology may aid in understanding these complex concepts.

In 2014, Norton, Hamel, and Brodie at the Kaiser Family Foundation conducted a large-scale assessment of Americans' familiarity with health insurance terms and concepts by surveying 1,292 U.S. adults. Overall, $52 \%$ of the public were able to answer 7 out of 10 questions correctly. However, $28 \%$ answered four or fewer questions correctly, $8 \%$ gave no correct answers, and only

The International Journal of Information, Diversity, \& Inclusion, 3(4), 2019

ISSN 2574-3430, jps.library.utoronto.ca/index.php/ijidi/index

DOI: $10.33137 /$ ijidi.v3i4.33012 
$4 \%$ answered all 10 questions correctly. The questions that drew upon individuals' numeracy skills to calculate out-of-pocket expenses proved to be the most difficult. Demographically, individuals with lower levels of education, younger Americans, and the uninsured scored lower on the health insurance literacy quiz. Their findings of younger Americans scoring lower on health insurance literacy suggests the need for additional research in this area, as the majority of health insurance literacy research has not included this population in their studies and have instead identified older age as relating to lower health insurance literacy skills.

Relatedly, Wong et al. (2015) used semi-structured interview techniques to assess young adults' understanding of health insurance terms and to identify participants' perceived advantages and disadvantages of obtaining health insurance coverage. In their participant group of 33 young adults between the ages of 19 and 30, participants demonstrated poor health insurance literacy skills, with $48 \%$ incorrectly defining deductible and $78 \%$ incorrectly defining coinsurance. When the researchers asked participants to self-rate their ability to understand health insurance terminology, confidence was poorly correlated with true understanding of the concepts. Costsharing concepts, such as deductibles, coinsurance, and out-of-pocket maximums were particularly confusing for their group of participants.

\section{Readability of Health Insurance Materials}

In addition to broader measurements of health insurance literacy, researchers have also assessed the readability of health insurance materials and individuals' interactions with these resources. Yin et al. (2009) extracted data from the 2003 NAAL to explore the ability of parents of young children to fill out health insurance forms. In this population of 6,100 parents, $68.4 \%$ were unable to complete a health insurance form properly, and $65.9 \%$ were unable to calculate the annual cost of a health insurance policy on the basis of family size. Perhaps it follows logically that the parents with below-basic health literacy were more likely to have a child without health insurance in their household. The authors conclude that given the large proportion of U.S. parents with low health literacy, "decreasing literacy demands on parents, including simplification of health insurance and other medical forms . . . is needed to decrease healthcare access barriers for children and ... a ameliorate existing child health disparities" (p. S289).

Researchers have also assessed the reading levels of Medicaid and other health care plan applications. Pati et al. (2012) examined compliance of Medicaid-renewal applications to the established state reading level guidelines. As of 2008, 45 states had reading level guidelines for the Medicaid-related materials, yet $24(52.2 \%)$ of the states failed to meet their own guidelines on three readability tests (the Flesch-Kincaid Grade Level Index, developed by the U.S. Navy to indicate how difficult a passage is to understand; New Fog Count, a revised and easier-to-use version of the Flesch-Kincaid; and FORCAST, the only readability test not designed for running narrative and instead suitable for measuring the readability of forms). As the authors emphasize, "complying with established reading level guidelines for Medicaid-related materials is one simplification strategy that should be implemented to improve access" (p. 297).

Wallace, DeVoe, and Hansen (2011) conducted a more holistic assessment of Children's Health Insurance Program (CHIP) applications by assessing reading demands, layout characteristics, and document complexity. They assessed these characteristics of online English-language $(n=50)$ and Spanish-language $(n=39)$ Medicaid/CHIP enrollment applications through Lexile Analyzer (to assess reading demands), the User-Friendliness Tool (to assess layout), and the PMOSE/IKIRSCH scale (to assess document complexity). While the low-literacy guidelines state that applications

The International Journal of Information, Diversity, \& Inclusion, 3(4), 2019

ISSN 2574-3430, jps.library.utoronto.ca/index.php/ijidi/index

DOI: $10.33137 /$ ijidi.v3i4.33012 
should be written at a 6th grade or lower reading level and using a font of 12 points or larger, the results showed that the application "Signature" pages were written at a high school reading level and only five enrollment applications (5.6\%) consistently used a 12-point or larger font size throughout. Wallace et al. determined that document complexity was above recommended levels, with the majority of the applications ranking highly difficult. In addition, the authors noted that to increase access for all populations, every state should provide an online Spanishlanguage version of the Medicaid/CHIP enrollment application, whereas at the time of their study only 39 of the states provided a Spanish-language version.

To address these demonstrated gaps, Gazmararian, Beditz, Pisano, and Carreón (2010), comprising a team of researchers from Emory University and America's Health Insurance Plans, sought to develop a health literacy assessment tool for health plans. Gazmararian et al. designed the tool to serve as a benchmark to address the "magnitude and consequences of low health literacy ... . [and] the role health plans are playing and the activities they undertake to address this problem" (p. 93). The areas of focus were identified through discussion with health plan representatives and a brief survey of health plans. Through this work they proposed six main areas of evaluation: information for members/navigation, member services/communication, web navigation, forms, nurse call line, and nurse case/disease management. They conducted a pilot study of their assessment tool on eight health plans. After incorporating reactions from this pilot study, the researchers launched the full assessment tool in 2009 (Emory University, 2010).

Lawson, Carreón, Veselovskiy, and Escarce (2011) explored the role of culturally and linguistically appropriate services (CLAS) in health insurance literacy. They surveyed 123 health plans about collecting language preference data and determined that $74.0 \%$ of health plans collected language data on preferred and primary languages (commercial $60.0 \%$, Medicaid $89.1 \%$, Medicare 91.7\%). Nearly all of the health plans reported offering language services, including interpretation services via phone, multilingual informational handouts, and access to bilingual providers. The authors suggested that the "availability of a full range of culturally and linguistically appropriate health care services is essential for overcoming barriers and accessing timely care" (p. e479). Providing these types of services is one way in which health disparities might be addressed in populations in which English is not the native language.

Beyond challenges with the readability of materials, there may be a lack of appropriate information for specific populations that are more likely to face health disparities due to their health status. In Lindner, Rowland, Spurlock, Dorn, \& Davis' (2018) study of disability advocates, participants highlighted a lack of information to help people with disabilities understand specific plan characteristics and choose the most appropriate plan. In some reported cases, individuals with disabilities often selected plans that minimized monthly premiums without recognizing this would lead to higher copayments and, therefore, restricted health care access. Those creating health insurance informational materials should assess not only the readability of provided materials but also the information available for those in specific populations that may require specialized medical care.

\section{Proposing an Integrated Framework for Health Insurance Literacy}

There are currently two published models of health insurance literacy. McCormack et al.'s (2009) Conceptual Framework for Health Insurance Literacy focuses more on specific, individual background characteristics, such as health status and demographics (including age, education, race, and culture), that might be identified as traits attributed to differences in health insurance

The International Journal of Information, Diversity, \& Inclusion, 3(4), 2019

ISSN 2574-3430, jps.library.utoronto.ca/index.php/ijidi/index

DOI: $10.33137 /$ ijidi.v3i4.33012 
literacy skills (e.g., lower levels of education has been shown to be correlated with lower health insurance literacy levels). Paez et al.'s (2014) Health Insurance Literacy Conceptual Model includes more domain-specific tasks, such as completing health insurance forms, calculating cost-sharing, and other insurance-related skills to model the concept. Barnes, Hanoch, and Rice (2015) stress the multi-dimensionality of this topic, stating that health insurance literacy is "likely influenced by cognitive abilities consumers possess (e.g., numeracy) and the amount of information available in the decision environment" (p. 60). It may be necessary to combine multiple models to create a fuller picture of health insurance literacy, understanding both the individual characteristics as well as individual abilities that form an individual's health insurance literacy.

\section{McCormack et al.'s Conceptual Framework for Health Insurance Literacy}

Using data collected from 1,202 Medicare beneficiaries, McCormack et al. (2009) developed a conceptual framework for health insurance literacy to "integrate a range of health- and insurance-related variables" (p. 227). Their model includes factors such as health status, age, education, race, culture, financial literacy, numeracy, health literacy, and health care decisionmaking. The inclusion of demographic factors supports further research into the connections between aspects such as age, education, race, and culture and the ability to interact with health insurance materials. Since McCormack et al.'s conclusions were drawn from a population of older adults, only $12.7 \%$ of whom were under age 65 , further studies should be conducted to extend the implications to a wider group. The framework developed by McCormack et al. is novel in that it was the first to model health insurance literacy. In addition, it combines multiple facets, such as demographic factors, financial literacy, and health literacy, building on strong areas of research ripe for further exploration in a new context.

\section{Paez et al.'s Health Insurance Literacy Conceptual Model}

Paez et al. (2014) created a Health Insurance Literacy Conceptual Model which identifies knowledge, information seeking, document literacy, and cognitive skills as the four domains that impact individuals' health insurance literacy, with self-efficacy as an underlying domain. These domains were identified through a combination of a literature review, key informant interviews, and a stakeholder group of subject experts. They are operationalized in Paez et al.'s Health Insurance Literacy Measure, which is comprised of four scales: two designed to assess individuals' self-efficacy in choosing a health insurance plan and two that assess self-efficacy and past behavior navigating and using health insurance.

\section{Integrated Framework for Health Insurance Literacy}

In an effort to propose a framework that would represent the multi-faceted nature of this topic, the author created an Integrated Framework for Health Insurance Literacy (see Figure 1). This integrated framework includes the demographic variables explored in the research described in this literature review. It also combines existing relevant models: the two health insurance literacy models discussed above (McCormack et al.'s Conceptual Framework for Health Insurance Literacy [2009] and Paez et al.'s Health Insurance Literacy Conceptual Model [2014]) and two health insurance decision-making models (Klinkman's The Consumer's Choice of Health Care Plan Framework [1991] and Sainfort and Booske's Conceptual Framework of Consumer Selection of Health Plans [1996]).

The International Journal of Information, Diversity, \& Inclusion, 3(4), 2019

ISSN 2574-3430, jps.library.utoronto.ca/index.php/ijidi/index

DOI: $10.33137 /$ ijidi.v3i4.33012 
Klinkman's framework focuses on the relationships among guarantor (i.e., insurance companies), employer, and consumer. It includes the steps taken by employers who select between available options to determine what they will offer their employees. It also includes the interactions between the consumer, the menu of available choices selected by the employer, and the consumer's ultimate choice. Sainfort and Booske (1996) built on the work by Klinkman and other health insurance researchers to create a conceptual framework of consumer selection of health plans that features a hypothesized relationship between background variables, health plan choice elements, and health plan information. Both of these frameworks point to the important role that the health insurance plans themselves play in the process individuals undergo when making a choice between the plans available to them.

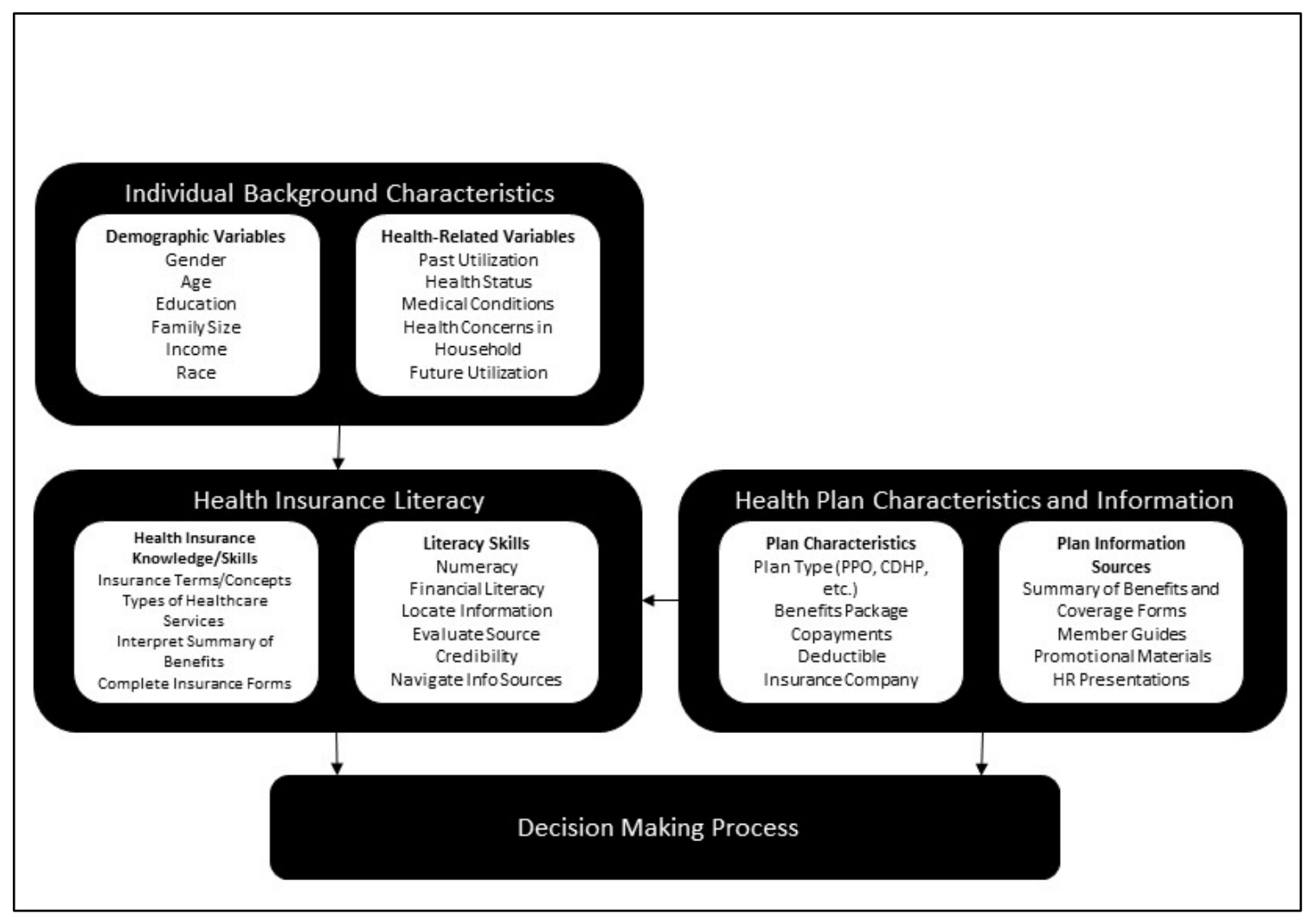

Figure 1. Integrated Framework for Health Insurance Literacy

This framework was created through identifying common characteristics in the models (e.g., demographic variables, health-related variables), as well as common relationships (e.g., relationship between background characteristics and literacy levels). The Integrated Framework begins with an individual's background characteristics, including demographic variables (identified in health insurance literacy research and previous models to play a role in an individual's abilities and skills) and health-related variables, including factors identified by previous research as playing a role in an individual's health insurance literacy skills such as previous health insurance utilization (Norton et al., 2014; Politi et al., 2014) and health status (McCormack et al., 2009).

The International Journal of Information, Diversity, \& Inclusion, 3(4), 2019

ISSN 2574-3430, jps.library.utoronto.ca/index.php/ijidi/index

DOI: $10.33137 /$ ijidi.v3i4.33012 
The Integrated Framework then demonstrates the relationship between an individual's traits (i.e., demographic and health-related variables) and their ability to use a health insurance literacy skill set, echoing the identified relationship between health disparities and health insurance literacy outlined in this literature review. That is, the framework proposes that an individual's own, specific background characteristics lead to their health insurance knowledge and skills and literacy skills. The specific inclusion of gender, age, education, and race is designed to encourage the continued exploration of these demographic variables and their relationship with health insurance literacy to identify potential sources of health disparities.

The framework also depicts the impact that available health plan characteristics and information play on an individual's ability to use their skills effectively. Health plan characteristics play both a direct role in the decision making process (as the health plan types and benefits packages are what individuals have to choose from) but also a role in the individual's ability to draw on their health insurance literacy knowledge and skills. For example, the presence of multiple plan types (i.e., multiple plan characteristics) may impact an individual's ability to use their health insurance literacy skill set; that is, it may be easier to compare between two preferred provider organizations (PPOs) and less feasible to compare between a PPO and a CDHP.

In addition, if an individual does not have access to helpful information sources, they may not be able to identify definitions and make the necessary summaries and comparisons to select an appropriate insurance choice. The inclusion of these aspects in the Integrated Framework is also supported by research on the role that the readability of insurance materials plays in health insurance literacy, explored earlier in this literature review. A potential area for librarians and information professionals to lend their expertise is to advocate for easy-to-read, multilingual insurance materials and to provide access to resources that assist individuals in understanding health insurance information and making selections between their available choices.

\section{Limitations}

This paper focuses on health insurance literacy research conducted in the United States. The majority of citizens in the U.S. face a high level of demand to make choices between a select number of options (either through Employer Sponsored Insurance or the Health Insurance Marketplace), making health care coverage in the U.S. different from many other developed countries. Thus, most health insurance literacy research has been conducted with a U.S. national focus. Health insurance literacy research conducted in other countries is outside of the scope of this particular paper as other health care systems place less demand on individuals to select between options and most often offer a national insurance coverage as a default option. More research is needed to compare health insurance literacy trends in other developed countries.

Health insurance choice research, discussed briefly here, operates on the assumption that there is a fiscally appropriate coverage option for individuals and then assesses whether they are able to identify which coverage option is most appropriate. This research may fail to recognize individual preferences for payment structures; some individuals may prefer to pay more money up front to cover all possible scenarios whereas others may wish to keep more of their paycheck and pay more when a health need arises. Relatedly, individuals have different risk tolerance levels, which most certainly also play a role when considering health insurance options. It should be acknowledged that health insurance literacy skills are larger than simply selecting the most fiscally appropriate coverage option from a list of choices.

The International Journal of Information, Diversity, \& Inclusion, 3(4), 2019

ISSN 2574-3430, jps.library.utoronto.ca/index.php/ijidi/index

DOI: $10.33137 /$ ijidi.v3i4.33012 
Lastly, not all individuals have a choice when it comes to health insurance. Individuals may have coverage through Medicare (which is limited by age eligibility), Medicaid (limited by income), and other sources (e.g., a single option through their employer) that prohibit the opportunity to select between available choices. Additional research should be conducted to explore health insurance literacy in individuals who are asked to navigate health insurance (such as enroll in and use coverage) without having a choice between different coverage options.

\section{Conclusion}

Understanding the variables that affect health insurance literacy is just the beginning. Health insurance literacy concerns also extend beyond selecting a health insurance coverage option. Once individuals are insured, their health insurance literacy levels may dictate how effectively they are able to navigate the health care system. For example, if an individual unwittingly uses an out-of-network physician or hospital, it could cost the person thousands of dollars more than selecting health care providers within the insurer's network. As Levitt (2015) underscores "the lack of health insurance literacy (and numeracy) has important implications for how effectively people use health care services and their insurance" (p. 556). For those with lower levels of income, the potential to pay larger amounts of money for coverage or care due to limited health insurance literacy skills compounds financial and health disparities.

Demographic differences in health insurance literacy skills have been explored in several studies (Federman et al, 2009; Kutner et al., 2006; McCormack et al., 2009; Politi et al., 2014) pointing to an opportunity to create targeted outreach to populations at highest risk of having lower health insurance comprehension and navigation skills, including racial and ethnic minorities, lower socio-economic status, age, and insurance coverage status. When considering these populations in tandem with populations more likely to be impacted by health disparities, the overlap indicates an opportunity for further research and exploration to better understand the connections between health insurance literacy and health disparities.

This literature review identified research that explored awareness of health insurance, measurements of health insurance literacy, and readability of health insurance materials. By focusing on the relationship between individual demographic backgrounds and health insurance literacy abilities, this review aimed to connect health disparities directly with health insurance literacy. The Integrated Framework for Health Insurance Literacy is proposed as a method for further studying the impact that demographic factors have on health coverage and health status through individuals' health insurance literacy skills.

\section{References}

Barnes, A. J., Hanoch, Y., \& Rice, T. (2015). Determinants of coverage decisions in health insurance marketplaces: Consumers' decision-making abilities and the amount of information in their choice environment. Health Services Research, 50(1), 58-80. https://doi.org/10.1111/1475-6773.12181

Courtemanche, C. J., \& Zapata, D. (2014). Does universal coverage improve health? The Massachusetts experience. Journal of Policy Analysis and Management, 33(1), 36-69. http://dx.doi.org/10.1002/pam.21737

The International Journal of Information, Diversity, \& Inclusion, 3(4), 2019

ISSN 2574-3430, jps.library.utoronto.ca/index.php/ijidi/index

DOI: $10.33137 /$ ijidi.v3i4.33012 
Emory University. (2010). Health plan organizational assessment of health literacy activities. Retrieved from https: //ahip.org/wpcontent/uploads/2017/01/HealthPlanOrganizationalAssessmentofHealthLiteracyActiviti es.pdf

Federman, A. D., Safran, D. G., Keyhani, S., Cole, H., Halm, E. A., \& Siu, A. L. (2009). Awareness of pharmaceutical cost-assistance programs among inner-city seniors. The American Journal of Geriatric Pharmacotherapy, 7(2), 117-29. https://doi.org/10.1016/j.amjopharm.2009.04.003

Gazmararian, J. A., Beditz, K., Pisano, S., \& Carreón, R. (2010). The development of a health literacy assessment tool for health plans. Journal of Health Communication, 15(Supplement 2), 93-101. https://doi.org/10.1080/10810730.2010.499986

Kaiser Family Foundation. (2008). How private health coverage works: A primer. Retrieved from http://kff.org/health-costs/issue-brief/how-private-health-coverage-works-aprimer/

Kaiser Family Foundation. (2016). Health insurance coverage of the total population. Retrieved from http://kff.org/other/state-indicator/total-population/

Kim, J., Braun, B., \& Williams, A. D. (2013). Understanding health insurance literacy: A literature review. Family and Consumer Sciences Research Journal, 42(1), 3-13. https://doi.org/10.1111/fcsr.12034

Klinkman, M. S. (1991). The process of choice of health care plan and provider: Development of an Integrated Analytic Framework. Medical Care Review, 48(3), 295-330. https://doi.org/10.1177\%2F002570879104800304

Kutner, M., Greenburg, E., Jin, Y., \& Paulsen, C. (2006). The health literacy of America's adults: Results from the 2003 National Assessment of Adult Literacy (NCES 2006-483). Washington, DC: National Center for Education Statistics.

Lawson, E. H., Carreón, R., Veselovskiy, G., \& Escarce, J. J. (2011). Collection of language data and services provided by health plans. The American Journal of Managed Care, $17(12)$, e479-87.

Levitt, L. (2015). Why health insurance literacy matters. The Journal of the American Medical Association, 313(6), 555-556. doi:10.1001/jama.2014.17419

Lindner, S., Rowland, R., Spurlock, M., Dorn, S., \& Davis, M. (2018). "Canaries in the mine..." the impact of Affordable Care Act implementation on people with disabilities: Evidence from interviews with disability advocates. Disability and Health Journal, 11(1), 86-92. https: //doi.org/10.1016/j.dhjo.2017.04.003

Loewenstein, G., Friedman, J. Y., McGill, B., Ahmad, S., Linck, S., Sinkula, S., . . . \& Volpp, K. G. (2013). Consumers' misunderstanding of health insurance. Journal of Health Economics, 32(5), 850-62. https://doi.org/10.1016/j.jhealeco.2013.04.004

McCormack, L., Bann, C., Uhrig, J., Berkman, N., \& Rudd, R. (2009). Health insurance literacy

The International Journal of Information, Diversity, \& Inclusion, 3(4), 2019

ISSN 2574-3430, jps.library.utoronto.ca/index.php/ijidi/index

DOI: $10.33137 /$ ijidi.v3i4.33012 
of older adults. Journal of Consumer Affairs, 43(2), 223-248.

https://doi.org/10.1111/j.1745-6606.2009.01138.x

Miller, V. M. (2007). Poor eHealth literacy and consumer-directed health plans: A recipe for market failure. The American Journal of Bioethics, 7(11), 20-22.

https://doi.org/10.1080/15265160701638587

Norton, M., Hamel, L., \& Brodie, M. (2014). Assessing Americans' familiarity with health insurance terms and concepts. Retrieved from http: / / kff.org/health-reform/pollfinding/assessing-americans-familiarity-with-health-insurance-terms-and-concepts/

Paasche-Orlow, M. K., Parker, R. M., Gazmararian, J. A., Nielsen-Bohlman, L. T., \& Rudd, R. (2005). The prevalence of limited health literacy. Journal of General Internal Medicine, 20(2), 175-184. https://dx.doi.org/10.1111\%2Fj.1525-1497.2005.40245.x

Paez, K.A., Mallery, C.J., Noel, H., Pugliese, C., McSorely, V.E., Lucado, J.L., \& Ganachari, D. (2014). Development of the Health Insurance Literacy Measure (HILM): Conceptualizing and measuring consumer ability to choose and use private health insurance. Journal of Health Communication, 19 (Supplement 2), 225-239. https://dx.doi.org/10.1080\%2F10810730.2014.936568

Pati, S., Kavanagh, J. E., Bhatt, S. K., Wong, A. T., Noonan, K., \& Cnaan, A. (2012). Reading level of Medicaid renewal applications. Academic Pediatrics, 12(4), 297-301. https://doi.org/10.1016/j.acap.2012.04.008

Patient Protection and Affordable Care Act, Public Law 148, 111th Cong., 2nd sess. (2010).

Politi, M.C., Kaphingst, K.A., Kreuter, M., Shacham, E., Lovell, M.C., McBride, T. (2014). Knowledge of health insurance terminology and details among the uninsured. Medical Care Research and Review, 71(1), 85-98. https://doi.org/10.1177/1077558713505327

Sainfort, F. \& Booske, B. C. (1996). Role of information in consumer selection of health plans. Health Care Financing Review, 18(1), 31-54.

Thorlby, R. \& Arora, S. (2019). The English health care system. The Commonwealth Fund. Retrieved from https://international.commonwealthfund.org/countries/england/

U.S. Department of Health and Human Services. (2001). Mental health: Culture, race, and ethnicity: A supplement to mental health, a report of the Surgeon General. Rockville, MD: U.S. Public Health Service.

U.S. Department of Health and Human Services. (2008). America's health literacy: Why we need accessible health information (issue brief). Retrieved from

https://health.gov/communication/literacy/issuebrief/

Vardell, E. (2013). Readability levels of health insurance Summary of Benefits and Coverage forms. Paper presented at the Annual Conference of the Medical Library Association, Boston, MA. Retrieved from https://www.ncbi.nlm.nih.gov/pmc/articles/PMC3878945/

Vardell, E. J. (2017). Health insurance literacy: How people understand and make health

The International Journal of Information, Diversity, \& Inclusion, 3(4), 2019

ISSN 2574-3430, jps.library.utoronto.ca/index.php/ijidi/index

DOI: $10.33137 /$ ijidi.v3i4.33012 
insurance purchase decisions (Doctoral dissertation). Available from ProQuest Dissertations \& Theses Global. (10283966)

Wallace, L. S., DeVoe, J. E., \& Hansen, J. S. (2011). Assessment of children's public health insurance program enrollment applications: A health literacy perspective. Journal of Pediatric Health Care: Official Publication of National Association of Pediatric Nurse Associates \& Practitioners, 25(2), 133-137. https://doi.org/10.1016/j.pedhc.2010.11.009

Wong, C. A., Asch, D. A., Vinoya, C. M., Ford, C. A., Baker, T., Town, R., \& Merchant, R. M. (2015). Seeing health insurance and HealthCare.gov through the eyes of young adults. Journal of Adolescent Health, 57(2), 137-143.

https://doi.org/10.1016/j.jadohealth.2015.04.017

Yin, H. S., Johnson, M., Mendelsohn, A. L., Abrams, M. A., Sanders, L. M., \& Dreyer, B. P. (2009). The health literacy of parents in the United States: A nationally representative study. Pediatrics, 124(Supplement 3), S289-298. https://doi.org/10.1542/peds.2009$\underline{1162 \mathrm{E}}$

Emily Vardell (evardell@emporia.edu) is an Assistant Professor in the School of Library and Information Management at Emporia State University. She teaches graduate courses on the foundations of library and information science, research methods, reference, consumer health, and health sciences librarianship. Her research interests are in the area of health information behavior with a focus on health insurance literacy and how people make health insurance decisions. Dr. Vardell earned her PhD from the School of Information and Library Science (SILS) at the University of North Carolina at Chapel Hill in 2017 and her Master of Library Science degree from Texas Woman's University in 2007. Dr. Vardell has received grants from the Medical Library Association and the National Network of Libraries of Medicine, been awarded the Beta Phi Mu Eugene Garfield Doctoral Dissertation Fellowship, and served as a Fulbright scholar in Vienna, Austria.

The International Journal of Information, Diversity, \& Inclusion, 3(4), 2019

ISSN 2574-3430, jps.library.utoronto.ca/index.php/ijidi/index

DOI: $10.33137 /$ ijidi.v3i4.33012 